Bio - grafía. Escritos sobre la Biología y su Enseñanza. ISSN 2027-1034

Edición Extraordinaria. p.p. 1433 - 1442

Memorias del IX Encuentro Nacional de Experiencias en Enseñanza de la Biología y la Educación Ambiental. IV Congreso Nacional de Investigación en Enseñanza de la Biología.

\title{
EDUCACIÓN SEXUAL Y PARA LA SALUD, UNA PROPUESTA DESDE LA PERSPECTIVA BIOPSICOSOCIAL EN NEIVA, HUILA.
}

\section{SEXUAL AND HEALTH EDUCATION, A PROPOSAL FROM THE BIOPSICOSOCIAL PERSPECTIVE IN NEIVA, HUILA.}

\author{
Cedeño Puentes, Katherine \\ Barrero Barrera, Francy Eliana \\ Mosquera, Jonathan Andrés ${ }^{1}$
}

\section{RESUMEN}

La educación para la salud en las comunidades educativas en Colombia, es un elemento que ha sido poco explorado, evidenciando que la educación sexual y sus componentes, han sido relegados al área de las ciencias naturales y en algunas ocasiones a los departamentos de psicopedagogía, enmarcando todo el proceso a las funciones reproductivas y los riesgos, dejando de lado el contexto y la promoción de la salud física, mental y cultural. Presentamos el planteamiento de una propuesta de investigación, en torno a la caracterización de las actitudes, los conocimientos y las prácticas en materia de Educación Sexual y Reproductiva, que se vienen adelantando al interior de tres Instituciones Educativas oficiales del municipio de Neiva. El trabajo se enmarca en un estudio de tipo mixto, en donde tomamos elementos del enfoque cualitativo para interpretar concepciones del estudiantado y profesorado, al tiempo se tienen en cuenta aspectos cuantitativos, apoyando algunos análisis descriptivos desde instrumentos estandarizados y de tipo escalamiento Likert. La revisión de antecedentes y del contexto en visitas preliminares, nos permite plantear que el estudiantado presenta bastante interés por abordar estas temáticas en el aula, particularizando en métodos anticonceptivos y las posibles prácticas saludables para el control de una vida sexual con responsabilidad. Sin embargo en los postulados de los docentes de ciencias naturales, es notorio el temor a hablar sobre el tema, dado que conllevan culturalmente rasgos conservadores y la falta de conocimientos actualizados, inciden en la evasión y la preferencia de dejar la unidad reducida a la función de reproducción que se desarrolla en grado octavo.

PALABRA CLAVE: Educación para la salud, concepciones, practicas, sexualidad

\begin{abstract}
${ }^{1}$ Grupo de Investigación Conocimiento Profesional del Profesor de Ciencias
\end{abstract}

Education for health in educational communities in Colombia is an element that has been little explored, evidencing that sexual education and its components have been relegated to the area of natural sciences and in some cases to the departments of psychopedagogy, framing The whole process to the reproductive functions and the risks, leaving aside the 


\title{
Bio - grafía. Escritos sobre la Biología y su Enseñanza. ISSN 2027-1034
}

\section{Edición Extraordinaria. p.p. 1433 - 1442}

\author{
Memorias del IX Encuentro Nacional de Experiencias en Enseñanza de la Biología y la \\ Educación Ambiental. IV Congreso Nacional de Investigación en Enseñanza de la \\ Biología.
}

context and the promotion of the physical, mental and cultural health. We present the proposal of a research proposal, about the characterization of attitudes, knowledge and practices in the field of Sexual and Reproductive Education, which are being advanced within three official educational institutions of the municipality of Neiva. The work is part of a study of mixed type, where we take elements of the qualitative approach to interpret conceptions of students and teachers, while taking into account quantitative aspects, supporting some descriptive analysis from standardized instruments and Likert scale type. The background and context review in preliminary visits, allows us to state that the students have a lot of interest in addressing these issues in the classroom, particularizing contraceptive methods and possible healthy practices for the control of a responsible sexual life. However, in the postulates of natural science teachers, it is notorious the fear to speak about the subject, since they carry culturally conservative traits and the lack of up-to-date knowledge, they influence in the evasion and the preference to leave the unit reduced to the function Of reproduction that develops in eighth degree.

KEY WORD: Education for health, conceptions, practices, sexuality

\section{INTRODUCCION}

La educación sexual es uno de los temas más comunes del siglo XXI donde las personas a pesar de que estamos en tiempos modernos no logran asimilar abiertamente la importancia que éste tiene para la sociedad. Así mismo es un término que puede tener muchos puntos de vista desde diferentes enfoques, es así, que para esta investigación queremos definirla como la orientación que se recibe en el transcurso de las etapas de vida de cada ser humano las cuales pueden ser transformadas a través de los tiempos según la sociedad, la familia y el colegio desde la parte biológica, psicológica, fisiológica y cultural es decir, desde un enfoque biopsicosocial. Según Conill (2005), la educación sexual constituye un complejo fenómeno social, el cual es inmerso al individuo que integra armónicamente el desarrollo de su personalidad, permitiéndole la asimilación de patrones históricos y culturales; es por ello, que se encuentra estrechamente ligada al contexto social, familiar y escolar porque desde allí se conforma, educa y evalúa la sexualidad desde distintos niveles de desempeño, interacción y comunicación que en ellos se establecen.

El Ministerio de Salud y Protección social diseña unos lineamientos nacionales para la aplicación y el desarrollo de estrategias de entornos saludables. En Colombia la Estrategia Escuelas Promotoras de la Salud se adoptó con el nombre de "Escuela Saludable" y fue acogida en 1997 por el Ministerio de Salud, hoy de la Protección Social, para liderarla en el nivel nacional. A comienzos de 1999, los Ministerios de Salud y Educación Nacional, el Instituto Colombiano de Bienestar Familiar - ICBF, y la OPS/OMS. La estrategia busca que mediante la acción comunitaria y el trabajo intersectorial se provea de mejores condiciones de vivienda y de salud a las personas, lo cual implica convocar a la integración otras instituciones y sectores para realizar un trabajo articulado 


\title{
Bio - grafía. Escritos sobre la Biología y su Enseñanza. ISSN 2027-1034
}

\section{Edición Extraordinaria. p.p. 1433 - 1442}

\author{
Memorias del IX Encuentro Nacional de Experiencias en Enseñanza de la Biología y la \\ Educación Ambiental. IV Congreso Nacional de Investigación en Enseñanza de la \\ Biología.
}

y planificado que permita responder a los compromisos de trabajo interinstitucional, intersectorial, interdisciplinario y de desarrollo comunitario.

En el 2000 el Ministerio de Salud, hoy Ministerio de la Protección Social, establece como una de las prioridades el desarrollo de proyectos para fortalecer la Estrategia Escuela Saludable en los departamentos y municipios en el marco de los Planes de Atención Básica - PAB. De esta manera en cooperación con otros organismos, el Ministerio de Educación Nacional viene desarrollando un proyecto piloto en el tema de la 'Educación para la Sexualidad y Construcción de Ciudadanía' y, a partir de 2007, se empezó a llevar a cabo un proyecto piloto específicamente para la estrategia de Estilo de Vida Saludable, que incorpora en primer lugar la Escuela Saludable.

De esta manera, la Organización Mundial para la Salud (OMS) propone cinco estrategias para desarrollarse en las instituciones educativas la enseñanza de la salud en educación sexual como: desarrollo de habilidades personales, fomento de interacción escuela-familia y comunidad, creación de un entorno saludable, reorientación e innovación de prácticas educativas y mejora de políticas normativas escolares que garanticen salud y bienestar a la comunidad educativa, favoreciendo que el estudiante alcance resultados positivos en el ámbito educativo y formativo.

De esta manera, el papel que juega la escuela, como escenario formativo que gesta la cultura del ciudadano, es clave, porque permite y favorece el desarrollo de la identidad saludable y responsable, o inciden en los hábitos que deterioren la calidad de vida. Según Gavidia (2016), una institución educativa saludable es o son aquellas que dotan de valores, habilidades y motivaciones para promover el bienestar de los alumnos; permitiéndoseles disfrutar de una salud autónoma, solidaria y gozosa, siendo dichos aspectos un recurso útil para la vida.

No obstante y a pesar de todos los mitos y tabúes que el tema ha podido generar en el país y a nivel general en las últimas décadas en el mundo, las instituciones educativas, son esenciales en el proceso de enseñanza y aprendizaje, pues como segundo hogar y escenario en donde el estudiantado pasa mayor parte del tiempo, es un lugar idóneo para el encuentro, la liberación, el intercambio de saberes y para hacer valer los derechos y asumir las responsabilidades. Es por esto que las Instituciones Educativas se convierten en la primera fuente en información sobre educación sexual; pretendiendo que los estudiantes reflejen en su vida el respeto, la toma de decisiones, la igualdad, y sobre todo la libre expresión y actuación de los demás sin importar las conductas que la sociedad pueda inculcar o denigrar en algún momento.

Este panorama, ha incidido en que la educación sexual se haya venido enseñando de manera muy limitada y poco apropiada debido a las concepciones que tiene la sociedad, basándose solamente a fundamentos teóricos limitados por diferentes situaciones que enmarcan una barrera que no permite llegar más a fondo a las practicas donde los estudiantes puedan sentirse identificados, es decir, verdaderam ente satisfechos con lo que quieren aprender. Según Soriano et al, (2013), la enseñanza de la educación sexual enfocada hacia la educación para la salud, debe priorizar no solo en los fundamentos biológicos y de prevención, sino que estos deben estar a la revisión de antecedentes y 


\title{
Bio - grafía. Escritos sobre la Biología y su Enseñanza. ISSN 2027-1034
}

Edición Extraordinaria. p.p. 1433 - 1442

\author{
Memorias del IX Encuentro Nacional de Experiencias en Enseñanza de la Biología y la \\ Educación Ambiental. IV Congreso Nacional de Investigación en Enseñanza de la \\ Biología.
}

concepciones del estudiantado, esperando incidir y potenciar la parte emocional, sentimental, actitudinal, y de conducta responsable.

Sin embargo, los maestros asumen que hablar de estos temas abiertamente es solo responsabilidad de los padres, limitándose a dar la clase de reproducción, fecundación, genitalidad, sin abordar algunos elementos culturales y posiblemente sociales. Para Soriano y Gonzales (2013), entonces la sexualidad en nuestra cultura está sujeta a miedos, ignorancias y mitos infundados que afectan a padres, educadores y políticos, etc., por ello resulta difícil una educación formal en materia de sexualidad en la escuela.

De esta manera, se hace entonces relevante establecer un proceso dialógico entre las partes del proceso educativo, en donde el eje sea la prevalecía de una cultura saludable que vincule elementos de la sociedad, garantizando la promoción de posturas responsables, es decir desarrollar una postura biopsicosocial. Esta investigación, se desarrolla al interior de dos Instituciones Educativas del sector oficial de Neiva, con el objetivo de caracterizar los conocimientos, las actitudes y las prácticas en educación sexual y reproductiva desde la perspectiva de los formadores y los estudiantes del grado octavo de dos Instituciones Educativas públicas del municipio de Neiva.

\section{METODOLOGÍA}

Esta investigación se desarrolla desde un enfoque mixto (cualitativo y cuantitativo), fundamentado desde el enfoque cuantitativo en un diseño no experimental transeccional o transversal de tipo descriptivo y exploratorio, y en el enfoque cualitativo desde un diseño en paralelo. El diseño mixto se fundamenta en la triangulación de métodos (Hernández, Fernández y Baptista, 2006). Es decir, es un proceso que recolecta, analiza y vincula datos cuantitativos y cualitativos en un mismo estudio para responder a un mismo problema (Teddllie y Tashakkori, 2003; Creswell, 2005; Mertens, 2005; Williams, Unrau y Grinnell, 2005). En este estudio, no se hará variar en forma intencional las variables independientes para ver su efecto sobre otras variables, correspondiendo al enfoque no experimental, donde la técnica será la observación de los fenómenos en su contexto natural (aula de clase, Institución Educativa, relación docente-estudiante), para después analizarlos (Kerlinger \& Lee, 2002).

Por otro lado el método de análisis para los datos cualitativos, será el análisis de contenido, el cual según Amórtegui (2011), es un método para estudiar y analizar las comunicaciones de forma sistemática, trata de analizar y estudiar con detalle el contenido de una comunicación escrita, oral y visual. Así mismo, se hará uso de la observación participante durante el tiempo de intervención en el aula, en el acompañamiento a estudiantes y en las jornadas de capacitación y sensibilización con los profesores.

Dentro de los instrumentos para la toma de datos, se empleará el cuestionario estandarizado por el Centro Latinoamericano de Perinatología - CLAP y la Organización Panamericana para la Salud - OPS, para procesos en educación sexual y reproductiva, con fines de caracterizar a los estudiantes de grado octavo de las dos Instituciones Educativas, al inicio y al final. A partir de este instrumento, se ha diseñado una entrevista 


\section{Bio - grafía. Escritos sobre la Biología y su Enseñanza. ISSN 2027-1034}

Edición Extraordinaria. p.p. 1433 - 1442

Memorias del IX Encuentro Nacional de Experiencias en Enseñanza de la Biología y la

Educación Ambiental. IV Congreso Nacional de Investigación en Enseñanza de la Biología.

semiestructurada, que se aplicará a los docentes, para recabar en conocimientos, actitudes y prácticas sobre el tema de estudio. Con apoyo de estudiantes de los programas de enfermería y educación física, se ha diseñado una secuencia didáctica que pretende abordar los contenidos temáticos de manera amena para los adolescentes y jóvenes en el aula de clases, permitiendo vincular al proceso elementos sociales, culturales, psicológicos y cognitivos, mediante talleres y capacitaciones.

Las dos (2) Instituciones Educativas se encuentran ubicadas en diferentes zonas urbanas de la ciudad, pertenecientes a los estratos socioeconómicas 1, 2 y 3. La población a intervenir son estudiantes de grado octavo los cuales oscilan en edades de 12 a 14 años; encontrándose en la etapa de adolescencia. Igualmente, trabajaremos con formadores, orientadores y directivos los cuales se encuentran inmersos al contexto de los estudiantes y han logrado tener un acercamiento hacia ellos.

\section{RESULTADOS}

\section{Principales Antecedentes}

La revisión bibliográfica hasta la fecha, permite evidenciar que los estudios en torno al tema de investigación, ha sido abordado en ocasiones, pero únicamente desde los aspectos biológicos de las poblaciones estudiadas, dejando de lado, los mitos y la incidencia de la cultura sobre el actuar de los jóvenes. De igual forma, es notorio el campo inexplorado en torno a la importancia de la escuela y de los formadores en la enseñanza y aprendizaje de una adecuada educación sexual, ya que no es solo designar la función a las Instituciones Educativas, sino analizar cómo se da el proceso y que elementos toman protagonismo dentro del mismo.

Dentro de los principales estudios a nivel internacional, nacional y regional que apuntan a la necesidad de un proceso articulado al interior de las instituciones educativas, entendiendo las perspectivas del estudiante y de los formadores, registra el trabajo de Cajiba (2013), se encuentra que la familia casi no hablan de la sexualidad con sus hijos y que la escuela lo realizan de manera muy superficial, sin embargo con las capacitaciones ellos logran comprender la importancia de estos temas para su vida. Así mismo Santos y Carvalho (2011), realizaron una investigación sobre las estrategias de enseñanza de educación sexual en la formación los profesores de ciencias y biología, encontrando que al realizarse estrategias metodológicas para una mejor enseñanza de educación sexual a profesores de ciencias naturales es muy satisfactoria, ya que varios testimonios de los estudiantes mostraron que las intervenciones fueron positivas, para la formación del profesional y una mejor enseñanza para una prevención de un embarazo precoz o no planificado por parte del adolescente.

En el plano nacional, se encontró que los adolescentes están muy interesados por su cuerpo y de conocer más a fondo sobre la sexualidad, pero sus padres prefieren guardar silencio por considerar estos temas como un tabú, este desconocimiento es lo que lo llevará reflejado en su práctica sexuales futuras (Cardona, Arboleda y Rosero, 2013). 


\author{
Bio - grafía. Escritos sobre la Biología y su Enseñanza. ISSN 2027-1034 \\ Edición Extraordinaria. p.p. 1433 - 1442 \\ Memorias del IX Encuentro Nacional de Experiencias en Enseñanza de la Biología y la \\ Educación Ambiental. IV Congreso Nacional de Investigación en Enseñanza de la \\ Biología.
}

Finalmente en el contexto regional hay muy pocas investigaciones realizadas respecto a la educación sexual, destacamos a Archury (1997), quien trabajó en torno al uso adecuado de métodos anticonceptivos en el contexto de una cultura de la sexualidad humana responsable por parte de los adolescentes, para prevenir los riesgos que implican un embarazo en esta etapa de la vida. Frente a este panorama, es menester informar que al interior del programa de Licenciatura en Ciencias Naturales: Física, Química y Biología, no se hallaron trabajos en relación al tema, a pesar de ser este el único programa de formación de maestros de ciencias naturales en el Huila, y teniendo como precedente, el hecho de que el Ministerio de Educación Nacional - MEN, plantea desde los Estándares Básicos de Competencias (EBC) los contenidos sobre reproducción, tema al que se ha reducido la educación sexual, alternando con aspectos como planificación, ciclo menstrual, anticonceptivos e infecciones de transmisión sexual (ITS) dentro del currículo de las ciencias naturales.

\title{
Cuestionario
}

El cuestionario principal de la investigación, es una adaptación con el debido permiso del formulario creado por el CLAP (Centro Latinoamericano de Perinatología / Unidad de salud de la Mujer y Reproductiva (CLAP/SMR) de la Organización Panamericana de la Salud (OPS), este documento está estandarizado y avalado por expertos de la OMS (Organización Mundial de la Salud). El Centro Latinoamericano de Perinatología (CLAP), fue creado por un grupo de profesionales comprometidos con la salud de las madres y sus recién nacidos, liderados por el Prof. Dr. Roberto Caldeyro Barcia, en el año 1970, mediante un acuerdo entre el Ministerio de Salud Pública de Uruguay, la Universidad de la República y la Organización Panamericana de la Salud (OPS), Oficina Regional para las Américas de la Organización Mundial de la Salud (OMS).

El instrumento, se compone por una serie de preguntas enfatizadas a la historia del adolescente (Apellidos y nombres, Domicilio, Lugar de Nacimiento, entre otros); una consulta principal (Edad, Estado Civil, etc.); Antecedentes personales y familiares los cuales están enfatizados en el padecimiento de enfermedades, alergias, vacunas, uso de medicamentos, problemas psicológicos y legales. Otro aspecto es la familia (Con quien vive, nivel de instrucción de los padres, el diagrama familiar y algunos consumos de la vivienda).

Para entrar más a fondo sobre la vida del estudiante se le pregunta sobre el nivel de educación, la realización de alguna actividad extra como lo es algún trabajo, la vida social (Aceptación en la sociedad, pareja actual, amigos entre otras actividades); hábitos/ Consumos (Alimentación adecuada, tabaco, alcohol, otras sustancias); aspectos ginecourológico (fecha de ultima menstruación, Ciclos regulares); Sexualidad (pareja sexual, edad inicio de relaciones sexuales, anticoncepción, entre otras) y situación psicoemocional y examen físico. 
Bio - grafía. Escritos sobre la Biología y su Enseñanza. ISSN 2027-1034

Edición Extraordinaria. p.p. 1433 - 1442

Memorias del IX Encuentro Nacional de Experiencias en Enseñanza de la Biología y la Educación Ambiental. IV Congreso Nacional de Investigación en Enseñanza de la Biología.

El cuestionario de igual forma, cuenta con el aval de la Unidad de Bioestadística y de Bioética de la Universidad Surcolombiana, permitiendo consolidar categorías de análisis que permitirán evaluar en un inicio, aspectos de la salud sexual y reproductiva de los jóvenes. De igual forma, el instrumento será aplicado al final de la intervención de aula.

\section{Construcción de entrevista semiestructurada}

A partir del cuestionario para los estudiantes, se construyeron veinte (20) preguntas orientadoras para la entrevista semiestructurada de los maestros en formación y ejercicio de las Instituciones Educativas. Las preguntas se presentan a continuación:

1. ¿Considera importante fomentar la educación para la salud en las Instituciones Educativas? ¿Por qué?

2. ¿Porque es importante la enseñanza de la educación sexual?

3. ¿Qué contenidos, considera usted relevantes e importantes para abordar en el aula, en relación a la educación sexual?

4. El entorno en que viven hoy nuestros adolescentes y jóvenes van enmarcados a las nuevas tecnologías incorporados al internet y a las redes sociales iconsideras que estos medios de comunicación son una buena fuente de información sobre la orientación en temas de sexualidad?

5. ¿Considera usted que sería esencial implementar en el currículo académico proyectos que promuevan el bienestar y la salud de la comunidad educativa?

6. ¿La educación sexual en las Instituciones Educativas, puede contribuir a generar bases oportunas para una apropiada vida saludable de los adolescentes y jóvenes en el departamento del Huila?

7. ¿De quién es la responsabilidad de inculcar la educación sexual en los adolescentes y jóvenes?

8. ¿Para usted es importante la enseñanza de la educación sexual para los estudiantes desde diferentes asignaturas?

9. ¿Cómo docente se siente preparado para abordar una temática sobre Educación Sexual en el aula con sus estudiantes?

10. ¿Los padres de familia han demostrado apoyo a esta iniciativa cómo aportan a la formación para la salud de sus hijos?

11. ¿Es función de los docentes, abordar temáticas de la educación para la salud y sexual con sus estudiantes?

12. Desde su experiencia docente, si estuviese abordando un tema sobre educación sexual con sus estudiantes ¿tendría en cuenta los saberes previos de cada estudiante? 
Bio - grafía. Escritos sobre la Biología y su Enseñanza. ISSN 2027-1034

Edición Extraordinaria. p.p. 1433 - 1442

Memorias del IX Encuentro Nacional de Experiencias en Enseñanza de la Biología y la

Educación Ambiental. IV Congreso Nacional de Investigación en Enseñanza de la Biología.

13. ¿utilizaría un modelo tradicional para enseñar estos temas o qué modelo implementaría para la enseñanza de la educación sexual?

14. ¿Cómo manejaría usted una situación en el aula de clase, si viera violencia de género o discriminación entre sus estudiantes?

15. ¿Considera que la enseñanza de la educación sexual en el aula, puede generar problemas e incidir en el comportamiento de manera deliberada de los adolescentes y jóvenes? Si su respuesta es afirmativa ¿Cuáles riesgos?

16. ¿Qué mitos o tabúes conoce en relación a la educación sexual en el aula?

17. ¿Qué papel juegan los saberes populares en el momento de enseñar temas sobre sexo, sexualidad y genero a los jóvenes?

18. ¿Qué beneficios traería abordar temáticas sobre educación sexual de manera clara y frecuente con los adolescentes y jóvenes?

19. ¿Creería usted que una institución educativa promotora de salud brindaría bienestar, permitiendo disfrutar de una salud autónoma, solidaria y gozosa vinculando a la realidad y a un contexto social en que viven los estudiantes actualmente?

20. ¿considera pertinente crear planes de mejoramiento sobre enseñanza de la Educación Sexual en las instituciones educativas?

Las preguntas orientadoras de la entrevista semiestructurada, han sido validadas por tres (3) expertos académicos en el área de la Educación para la Salud (Universidad Pedagógica Nacional) y Enseñanza de las Ciencias (Universidad de Almería - España).

\section{CONCLUSIONES PARCIALES}

La educación para la salud, es un tema poco abordado en la región sur colombiana, dejando profundos vacíos referenciales, evadiendo la responsabilidad que como formadores tenemos con nuestros adolescentes y jóvenes y su formación hacia una vida saludable y responsable, en donde se tomen decisiones oportunas y coherentes, por ejemplo sobre edad y mecanismos reproductivos, conductas y acciones responsables que permitan disfrutar las etapas vitales pero sin desencadenar acciones de riesgo para adolescentes y jóvenes.

Según la revisión bibliográfica, en el departamento del Huila, aún predominan concepciones alternas conservadoras en torno al sexo y la sexualidad, demostrando apatía hacia el tema en escenarios educativos y limitando las condiciones de enseñabilidad del tema, únicamente a los responsables de la salud (personal clínico), olvidando que las primeras fuentes de formación son la casa y la escuela. Por otro lado, es preocupante las estadísticas departamentales que nos arroja el DANE (Departamento Administrativo Nacional de Estadística) sobre índices de embarazos no deseados y en 
Bio - grafía. Escritos sobre la Biología y su Enseñanza. ISSN 2027-1034

Edición Extraordinaria. p.p. 1433 - 1442

Memorias del IX Encuentro Nacional de Experiencias en Enseñanza de la Biología y la

Educación Ambiental. IV Congreso Nacional de Investigación en Enseñanza de la Biología.

época de escolarización, donde el $20,5 \%$ de las mujeres con hijos en el país lo tuvieron entre los 15 y 19 años de edad, igualmente el número de ITS en jóvenes y las alarmas por acoso sexual, por causa de una mala educación oportuna y clara, que no se limite a propagandas y charlas, sino que sea constante, tangible y verificable, apreciando progresión en concepciones, actitudes y prácticas.

Es importante enfocar el estudio de la salud y el desarrollo de los adolescentes desde un perspectiva biopsicosocial, ya que la sexualidad humana es el resultado de la integración de tres dimensiones: biológica , social y psicológica; hay que abordarlo desde esa perspectiva porque en esta etapa es donde va enlazado el desarrollo y formación de la personalidad, atendiendo a nuestro sexo físico, haciendo referencia a nuestra emociones, sentimientos, pensamientos y demostrándolo en nuestras conductas y acciones.

La poca capacitación integral en educación sexual de adolescentes, se ve influenciada negativamente por la curiosidad, deseo y medios de comunicación que inducen a los jóvenes a realizar actividades sexuales a temprana edad acompañados de pasión y deseo, induciendo a la promiscuidad y pérdida del respeto por sí mismos, en total desconocimiento de las consecuencias de salud y carácter social que estas acciones conllevan.

\section{BIBLIOGRAFÍA:}

Amórtegui, E. (2011). Concepciones sobre prácticas de campo y su relación con el conocimiento profesional del profesor, de futuros docentes de Biología de la Universidad Pedagógica Nacional. Tesis de Maestría. Bogotá: Universidad Pedagógica Nacional.

Archury, B. (1997). Diagnóstico de niveles de información, actitudes y valores como base para el diseño de un programa de educación sexual respecto al control de natalidad, con y para los adolescentes del colegio cooperativo del municipio de Paicol, Huila. Tesis de grado, biblioteca Universidad Surcolombiana-Neiva.

Cajiba, R. (2013). La percepción de la sexualidad entre los adolescentes antes y después de participar en talleres educativos. Revista electrónica de enseñanza de las ciencias vol.12, $N^{\circ} 2,234-242$.

Cardona, J., Arboleda, P., y Rosero, C. (2013) Conocimientos actitudes y practicas acerca de las infecciones de trasmisión sexual en los adolescentes del municipio de caldas. Revista salud uninorte. Barranquilla-Colombia

Conill, J. (2005). Alternativa metodológica para concebir el proceso de educación de la sexualidad de los niños con retraso mental (Tesis para optar al título de doctor en Ciencias). Facultad de Biología, Universidad de La Habana, La Habana, Cuba. 
Bio - grafía. Escritos sobre la Biología y su Enseñanza. ISSN 2027-1034

Edición Extraordinaria. p.p. 1433 - 1442

Memorias del IX Encuentro Nacional de Experiencias en Enseñanza de la Biología y la

Educación Ambiental. IV Congreso Nacional de Investigación en Enseñanza de la Biología.

Creswell, J. (2005). Educational research: planning, conducting, and evaluating quantitative and qualitative Research (2 da Ed.). Upper Saddle River: pearson Education Inc.

Gavidia, V. (2016). Los ocho ámbitos de la educación para la salud en la escuela, departamento de didáctica de las ciencias experimentales y sociales, universidad de valencia.

Hernández, R., Fernández, C., y Baptista, P. (2006). Metodología de la investigación .Cuarta edición

Kerlinger, F.N. y Lee, H.B. (2002). Investigación del comportamiento: Métodos de investigación en ciencias sociales. México: McGraw-Hill Interamericana Editores

Ministerio de la Protección Social, Ministerio de Educación y Ministerio del Medio Ambiente Vivienda y Desarrollo Territorial (2006). Lineamientos nacionales para la aplicación y el desarrollo de las es. Recuperado de: https://www.minsalud.gov.co/sites/rid/Lists/BibliotecaDigital/RIDE/VS/PP/SA/lineami entos-nacionales-para-la-aplicacion-y-el-desarrollo-de-las-ees.pdf

Ministerio de Salud (2008). Servicios de salud amigable para adolescentes y jóvenes. Um modelo para adecuar las respuestas de los servicios de salud a las necesidades de adolescentes y jóvenes en Colombia. Segunda Edición, Bogotá.

Ministerio en Salud y Protección Social (2010). Política Nacional de sexualidad, derechos sexuales $y$ derechos reproductivos Colombia. Recuperado de: https://www.minsalud.gov.co/sites/rid/Lists/BibliotecaDigital/RIDE/DE/LIBRO\%20PO LITICA\%20SEXUAL\%20SEPT\%2010.pdf

Ministerio en Salud y Protección Social (2013). Compilación analítica de las normas de salud sexual y reproductiva en Colombia. Recuperado de: https://www.minsalud.gov.co/sites/rid/Lists/BibliotecaDigital/RIDE/VS/PP/compiladonormativa-salud-sexual-reproductiva.pdf

Organización Panamericana de la Salud (OPS). Consejería orientada en los jóvenes para prevenir VIH/ITS y para promover la salud sexual y reproductiva. Recuperado de: http://www1.paho.org/hq/dmdocuments/2009/consejeria\%20vih.pdf. (Visitada agosto $8,2017)$

Profamilia (2005). Encuesta Nacional de Demografía y Salud -2005- ENDS. Bogotá: Profamilia, p. 36.

Santos, N. y Carvalho (2011). Estrategias de enseñanza de educación sexual en la formación los profesores de ciencias y biología. Revista electrónica de enseñanza de las ciencias vol.10, №2,334-345.

Soriano, E., Gonzales, A. y Soriano, M. (2013). Educación para la salud sexual del enamoramiento al aborto, perfiles educativos vol. XXXVI, núm. 144,2014. 